
and Petra J. Kluger

\title{
Generation of an azide-modified extracellular matrix by adipose-derived stem cells using metabolic glycoengineering
}

\begin{abstract}
Natural extracellular matrix (ECM) represents an ideal biomaterial for tissue engineering and regenerative medicine approaches. For further functionalization, there is a need for specific addressable functional groups within this biomaterial. Metabolic glycoengineering (MGE) provides a technique to incorporate modified monosaccharide derivatives into the ECM during their assembly, which was shown by us earlier for the production of a modified fibroblast-derived dermal ECM. In this study, adipose-derived stem cells (ASCs) were treated with the azide-modified monosaccharide derivate 1,3,4,6-tetra-O-acetyl-N-azidoacetylgalactosamine

$\left(\mathrm{Ac}_{4} \mathrm{GalNAz}\right.$ ). Toxicity and viability assays after $24 \mathrm{~h}$ and 72 $\mathrm{h}$ incubation revealed high biocompatibility of $\mathrm{Ac}_{4} \mathrm{GalNAz}$ in contact with ASCs. The successful incorporation of the functional azide groups into the glycocalyx and the ECM of the ASCs was proven by conjugation with a fluorescent dye via a copper-catalyzed click reaction. Thus, $\mathrm{Ac}_{4} \mathrm{GalNAz}$ in combination with ASCs was confirmed to achieve an azidemodified ECM as a multifunctional biomaterial for further applications.
\end{abstract}

Keywords: Adipose-derived stem cells, clickECM, extracellular matrix, metabolic glycoengineering, azidemodified

https://doi.org/10.1515/cdbme-2019-0099

\footnotetext{
${ }^{*}$ Corresponding author: Svenja Nellinger: Reutlingen University, Alteburgstr 150, Reutlingen, Germany, e-mail: svenja.nellinger@reutlingen-university.de

Silke Keller, Alexander Southan: University of Stuttgart, Stuttgart, Germany

Valentin Wittmann: University of Konstanz, Konstanz, Germany Ann-Cathrin Volz, Petra J. Kluger: Reutlingen University,

Reutlingen, Germany
}

\section{Introduction}

The extracellular matrix (ECM) represents the natural environment of cells in an organism, wherein it is synthesized and assembled by tissue-specific cells. Next to native ECM, derived from decellularized mature tissues, cell-specific matrices can also be obtained from the in vitro culture of these cells. Varying with the specific tissue or cell source, the ECM contains variable amounts of collagens, other fibrous and nonfibrous proteins, proteoglycans and glycoproteins and exhibits different physical properties. Native and cell-derived ECMs are used and studied in a variety of applications as potential biomaterials such as cell-influencing coatings [1, 2], hybrid scaffolding materials for tissue engineering [3, 4], and bioinks $[5,6]$. A wide range of potential applications requires the modification of the ECM with specific addressable functional groups. Chemical modification of ECM compounds is challenging as it might affect matrix integrity. To address these issues, metabolic glycoengineering (MGE) represents a promising tool. This method is based on the incorporation of chemically modified monosaccharide derivatives into the natural intra- and extracellular oligosaccharide structures of the cell by its natural metabolic pathways [7, 8]. These functionalities can subsequently be addressed by bioorthogonal chemical ligation reactions $[9,10]$ for tuning chemical and physical properties and for visualization of glycoconjugates. Previously, we employed MGE with the azide-modified monosaccharide derivate 1,3,4,6-tetra-Oacetyl-N-azidoacetylgalactosamine $\left(\mathrm{Ac}_{4} \mathrm{GalNAz}\right)$ to generate a human fibroblast-derived dermal ECM containing azide groups [11] that can be addressed by copper-catalyzed azidealkyne cycloaddition [12, 13]. For this functionalized ECM (clickECM) we could show that the composition and functionality of the ECM was not affected by the MGE procedure and the modification with azide groups [11]. Later, also Gutmann et al. reported a similar approach using the corresponding azide-modified glucosamine derivative [14]. 
In this study, we aimed to prove whether the glycocalyx and ECM of adipose-derived stem cells (ASCs) can be modified with azide groups using $\mathrm{Ac}_{4} \mathrm{GalNAz}$ in MGE (Figure 1). Generation of an azide-modified stem cell ECM would be very promising for a wide range of applications especially in tissue engineering and regenerative medicine by representing a stem cell niche material with tunable chemical and physical properties. Such a unique biomaterial cannot be achieved by decellularization of tissues, as the stem cell niches are very small in vivo and integrated in matured neighboring tissues. Therefore, azide-modified ECM of ASCs provides multiple opportunities of further functionalization and application.

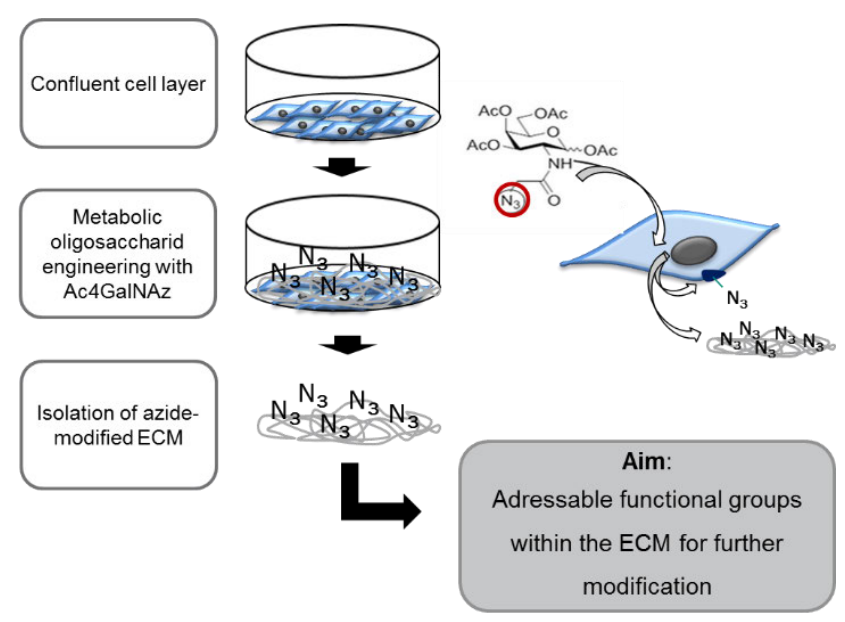

Figure 1: Schematic overview of the metabolic glycoengineering process with $\mathrm{ASCs}$ and $\mathrm{Ac}_{4} \mathrm{GaINAz}$. Cells were grown to confluency followed by the addition of $\mathrm{Ac}_{4} \mathrm{GalNAz}$. The incorporation of azide-groups into the glycocalyx and ECM occurs by MGE. Subsequently the azide-modified ECM was isolated by lysis of the cells. Generated azide-modified ECM could prospectively be used for further applications such as specific modifications of the ECM with molecules and for the use in tissue engineering and bioprinting approaches. (Modified after Ruff et al. [11])

\section{Materials and Methods}

\subsection{Isolation and expansion of ASCs}

ASCs were isolated from human tissue samples obtained from patients undergoing plastic surgery (Dr. Ziegler; Klinik Charlottenhaus, Stuttgart, Germany) as described before [15]. All research was carried out in accordance with the rules for investigation of human subjects as defined in the Declaration of Helsinki. Patients provided written agreement in compliance with the Landesärztekammer Baden-Württemberg (F-2012- 078, for normal skin from elective surgeries). ASCs were initially seeded at a density of $5 \times 10^{3}$ cells $\mathrm{cm}^{-2}$ in serumfree MSC growth medium (PELOBiotech, \#PB-C-MH-6750511-XF) containing $5 \%$ human platelet lysate.

\subsection{Biocompatibility assay}

The biocompatibility of $\mathrm{Ac}_{4} \mathrm{GalNAz}$ was evaluated by a lactate dehydrogenase (LDH) assay (TaKaRa Bio Inc. \#630117) and a resazurin assay (Sigma Aldrich, \#R7017). ASCs were treated with $100 \mu \mathrm{M} \mathrm{Ac} 4$ GalNAz or phosphate buffered saline (PBS) for $24 \mathrm{~h}$ and $72 \mathrm{~h}$. LDH assay was performed according to manufacturer's protocol with cell culture supernatant. For the resazurin assay, culture medium was changed to medium with resazurin salt $(11 \mu \mathrm{g} / \mathrm{mL})$ and incubated for $3 \mathrm{~h}$ at $37^{\circ} \mathrm{C}$ and 5 $\% \mathrm{CO} 2$.

\subsection{Detection of incorporated azide groups}

Incorporated azide-groups were detected via copper-catalyzed azide-alkyne cycloaddition using an alkyne-linked fluorophore as described by Ruff et al. [11]. Isolated paraformaldehyde-fixed ECM was stained according to the manufacturer's instructions. Cell nuclei were counterstained with $1 \mu \mathrm{g} / \mathrm{mL}$ 4',6-diamidino-2-phenylindole (DAPI, Serva Electrophoresis) in PBS for $10 \mathrm{~min}$ at RT. Fluorescence images (z-stacks) were taken using a Zeiss Axio Observer.

\subsection{Statistics}

All experiments were performed with cells from three different biological donors. Data was compared by a one-way analysis of variance and a Tukey post-hoc test using OriginPro 2018 b.

\section{Results and Discussion}

Biocompatibility of $\mathrm{Ac}_{4} \mathrm{GalNAz}$ was proven by $\mathrm{LDH}$ and resazurin assay (Figure 2). Control groups (Ctr) were incubated with PBS and absorption values were normalized to the control groups. LDH assay revealed no cytotoxic effects of $\mathrm{Ac}_{4}$ GalNAz after $24 \mathrm{~h}$ and $72 \mathrm{~h}$. 
A

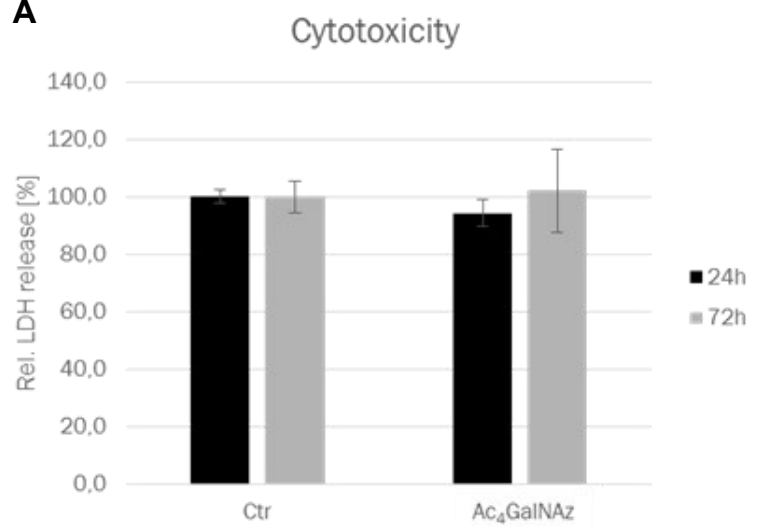

B

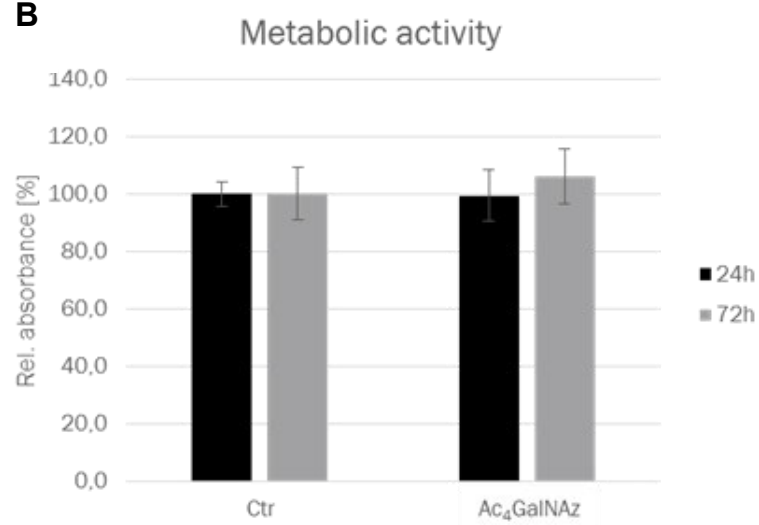

Figure 2: Biocompatibility of Ac4GalNAz in ACSs. ASCs were incubated with $100 \mu \mathrm{M}$ Ac4GalNAz for $24 \mathrm{~h}$ and $72 \mathrm{~h}$. Controls were treated with PBS. A: LDH Assay revealed no significant influence of azide-modified monosaccharide on cell death after $24 \mathrm{~h}$ and $72 \mathrm{~h}$. B: Resazurin assay showed no significant influence of azide-modified monosaccharide on metabolic activity after $24 \mathrm{~h}$ and $72 \mathrm{~h}$.

Metabolization of resazurin salt was comparable for cells treated with $\mathrm{Ac}_{4} \mathrm{GalNAz}$ to the control group. Thus, resazurin assay revealed no significant influence of $\mathrm{Ac}_{4} \mathrm{GalNAz}$ on metabolic activity of ASCs. These results are in line with previous studies using fibroblasts [11].

ECM of ASCs can be successfully isolated and harvested as thin sheet of protein network (Fig. $3 \mathrm{~A} ; \mathrm{i}$ ) or left as thin gellike film on the bottom of the petri dish (Figure. 3 A;ii). Incorporation of azide-groups into the glycocalyx and ECM of ASCs was proven by reaction with fluorophore-linked alkyne. In the untreated control group, no specific fluorescence staining was observed (Figure 3B). The cells treated with $\mathrm{Ac}_{4} \mathrm{GalNAz}$ exhibited a high specific fluorescence staining of the incorporated azide-groups. It was shown that azide groups were successfully incorporated into the glycocalyx of the cells. Moreover, for ECM isolated from ASCs and thereafter treated with $\mathrm{Ac}_{4} \mathrm{GalNAz}$, showed a specific fluorescence of stained azide groups. This confirmed the incorporation of $\mathrm{Ac}_{4} \mathrm{GalNAz}$ in the stem cell ECM. The azide groups were ubiquitously distributed in the glycocalyx and the ECM, comparable to the findings of Ruff et al. [11]
A
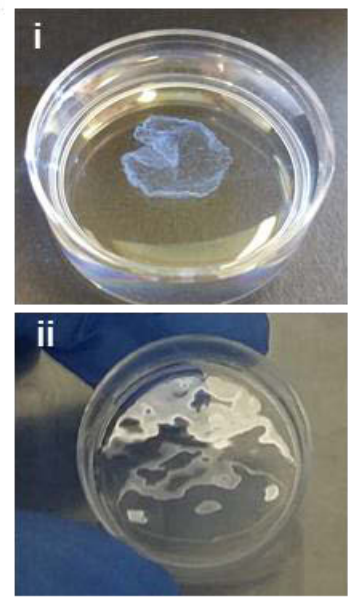

B
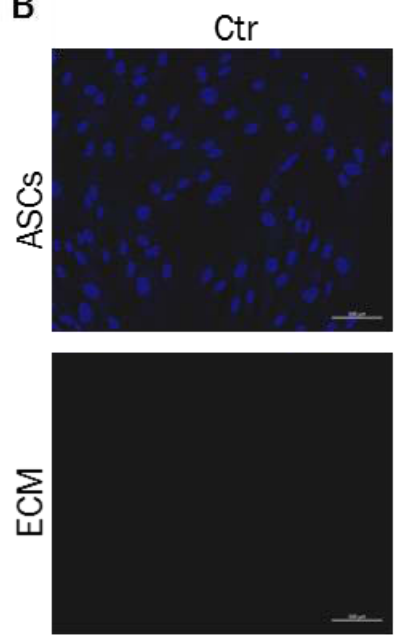

$\mathrm{Ac}_{4} \mathrm{GaINAz}$
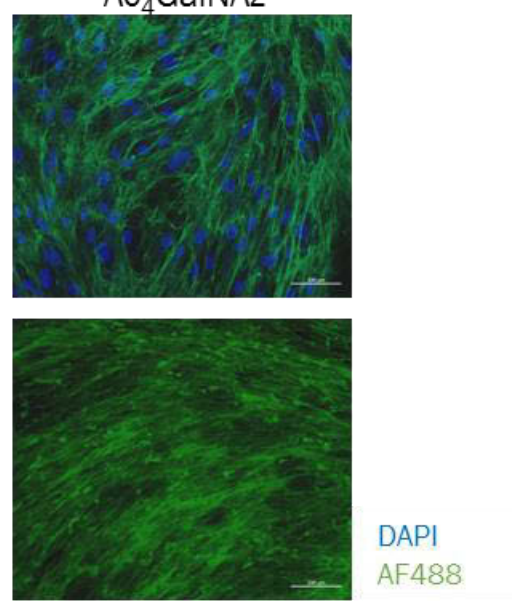

Figure 3: Characterization of ECM as biomaterial. A: Macroscopic pictures of isolated ECM. i: Isolated ECM floating in PBS. ECM can be isolated and collected as a gel-like sheet of a collagen network. ii: Isolated ECM on the bottom of a petri dish. After cell lysis and washing steps, the collagen network appears as a gel-like coating on the bottom of a petri dish $(\mathrm{d}=35 \mathrm{~mm})$ B: Fluorescence staining of the incorporated $\mathrm{Ac}_{4}$ GaINAz in ASCs and cell-derived ECM. Staining of the azide-groups shows the successful incorporation of azide groups into the glycocalyx and ECM of the ASCs. Negative controls (Ctr.) exhibit no fluorescence staining. Cell nuclei are counterstained with DAPI. (Scale bar: $100 \mu \mathrm{m}$ ) 


\section{Conclusion}

No negative impact on cell survival and metabolic activity of ASCs was detectable. Further, it was shown that stem cell ECM of ASCs can be successfully modified with azide groups, which can be further addressed by alkyne-groups via coppercatalyzed azide-alkyne cycloaddition. Based on these results, $\mathrm{Ac}_{4} \mathrm{GalNAz}$ may be considered a suitable azide-modified monosaccharide derivative to be used in MGE with ASCs. Thereby, this approach opens up a wide field of medical and material applications of ASC-derived ECM by yielding a multifunctional biomaterial with tuneable chemical and physical properties.

\section{Author Statement}

Research funding: We acknowledge the financial support of the Ministry of Science, Research and the Arts of BadenWürttemberg, Germany (\#33-7533-7-11.9/7/2).

Conflict of interest: Authors state no conflict of interest. Informed consent: Informed consent has been obtained from all individuals included in this study. Ethical approval: The research related to human use complies with all the relevant national regulations, institutional policies and was performed in accordance with the tenets of the Helsinki Declaration, and has been approved by the authors' institutional review board or equivalent committee.

\section{References}

[1] Guneta V, Loh QL, Choong C. Cell-secreted extracellular matrix formation and differentiation of adipose-derived stem cells in 3D alginate scaffolds with tunable properties. Journal of biomedical materials research Part A 2016;104:1090-101.

[2] Vafaei S, Tabaei SR, Guneta V, Choong C, Cho NJ. Hybrid Biomimetic Interfaces Integrating Supported Lipid Bilayers with Decellularized Extracellular Matrix Components. Langmuir 2018;34:3507-16.

[3] Flynn LE. The use of decellularized adipose tissue to provide an inductive microenvironment for the adipogenic differentiation of human adipose-derived stem cells. Biomaterials 2010;31:4715-24.

[4] Adam Young D, Bajaj V, Christman KL. Award winner for outstanding research in the PhD category, 2014 Society for Biomaterials annual meeting and exposition, Denver, Colorado, April 16-19, 2014: Decellularized adipose matrix hydrogels stimulate in vivo neovascularization and adipose formation. Journal of biomedical materials research Part A 2014;102:1641-51.

[5] Pati F, Jang J, Ha DH, Kim SW, Rhie JW, Shim JH, et al. Printing three-dimensional tissue analogues with decellularized extracellular matrix bioink. Nat Commun 2014;5.

[6] Pati F, Ha DH, Jang J, Han HH, Rhie JW, Cho DW. Biomimetic 3D tissue printing for soft tissue regeneration. Biomaterials 2015;62:164-75.

[7] Wratil PR, Horstkorte R, Reutter W. Metabolic Glycoengineering with N-Acyl Side Chain Modified Mannosamines. Angew Chem, Int Ed 2016;55:9482-512.

[8] Palaniappan KK, Bertozzi CR. Chemical Glycoproteomics. Chem Rev 2016;116:14277-306.

[9] Prescher JA, Bertozzi CR. Chemistry in living systems. Nat Chem Biol 2005;1:13-21.

[10] Sletten EM, Bertozzi CR. Bioorthogonal Chemistry: Fishing for Selectivity in a Sea of Functionality. Angew Chem, Int Ed 2009;48:6974-98.

[11] Ruff SM, Keller S, Wieland DE, Wittmann V, Tovar GEM, Bach $\mathrm{M}$, et al. clickECM: Development of a cell-derived extracellular matrix with azide functionalities. Acta biomaterialia 2017;52:159-70.

[12] Rostovtsev VV, Green LG, Fokin VV, Sharpless KB. A Stepwise Huisgen Cycloaddition Process: Copper(I)Catalyzed Regioselective "Ligation" of Azides and Terminal Alkynes. Angew Chem, Int Ed 2002;41:2596-9.

[13] Tornøe CW, Christensen C, Meldal M. Peptidotriazoles on Solid Phase: [1,2,3]-Triazoles by Regiospecific Copper(I)Catalyzed 1,3-Dipolar Cycloadditions of Terminal Alkynes to Azides. J Org Chem 2002;67:3057-64.

[14] Gutmann M, Braun A, Seibel J, Lühmann T. Bioorthogonal Modification of Cell Derived Matrices by Metabolic Glycoengineering. ACS Biomater Sci Eng 2018;4:1300-6.

[15] Huber B, Borchers K, Tovar GE, Kluger PJ. Methacrylated gelatin and mature adipocytes are promising components for adipose tissue engineering. Journal of biomaterials applications 2016;30:699-710. 